\title{
SPECIAL HOMOLOGICAL DIMENSIONS AND INTERSECTION THEOREM
}

\author{
TIRDAD SHARIF and SIAMAK YASSEMI*
}

\begin{abstract}
Let $(R, \mathfrak{m})$ be commutative Noetherian local ring. It is shown that $R$ is Cohen-Macaulay ring if there exists a Cohen-Macaulay finite (i.e. finitely generated) $R$-module with finite upper Gorenstein dimension. In addition, we show that, in the Intersection Theorem, projective dimension can be replaced by quasi-projective dimension.
\end{abstract}

\section{Introduction}

Throughout, the rings will denote a non-trivial, commutative, local, Noetherian ring and the modules are finite (that means finitely generated). Let $M$ and $N$ be finite $R$-modules and $\operatorname{pd}_{R} M<\infty$. The New Intersection Theorem of Peskine and Szpiro [13], Hochster [11], and P. Roberts [14], [15] yields an inequality

$$
\operatorname{dim}_{R} N \leq \operatorname{dim}_{R}\left(M \otimes_{R} N\right)+\operatorname{pd}_{R} M .
$$

By applying the inequality (1) with $N=R$ one derives,

$$
\operatorname{dim} R \leq \operatorname{dim}_{R} M+\operatorname{pd}_{R} M .
$$

By the Auslander-Buchsbaum Formula, the inequality (2) is equivalent to

$$
\text { cmd } R \leq \operatorname{cmd}_{R} M
$$

where $\operatorname{cmd}_{R} M=\operatorname{dim}_{R} M-\operatorname{depth}_{R} M$ is the Cohen-Macaulay defect of $M$ that is a non-negative integer which determines the failure of $M$ to be CohenMacaulay; we set cmd $R=\mathrm{cmd}_{R} R$.

In [3] Auslander and Bridger has generalized the notion of projective dimension to that of Gorenstein dimension. A finite $R$-module $M$ is said to be of G-dimension zero, and we write G-dim $\operatorname{dim}_{R} M=0$, if and only if

(1) $\operatorname{Ext}_{R}^{i}(M, R)=0$ for $i>0$.

\footnotetext{
* The second author is supported by a grant from IPM (NO. 82130212).

Received January 15, 2004; in revised form June 17, 2004.
} 
(2) $\operatorname{Ext}^{i}(\operatorname{Hom}(M, R), R)=0$ for $i>0$.

(3) The canonical map $M \rightarrow \operatorname{Hom}(\operatorname{Hom}(M, R), R)$ is an isomorphism.

For a non-negative integer $n$ the module $M$ is said to be of G-dimension at most $n$, and we write $\mathrm{G}-\operatorname{dim}_{R} M \leq n$, if and only if there exists an exact sequence

$$
0 \rightarrow G_{n} \rightarrow G_{n-1} \rightarrow \cdots \rightarrow G_{1} \rightarrow G_{0} \rightarrow M \rightarrow 0
$$

with G-dim $G_{R} G_{i}=0$ for all $0 \leq i \leq n$. In [17] Yassemi gave an example to show that the projective dimension can not replace by Gorenstein dimension in (1). On the other hand there are some other generalizations of projective dimension, i.e. complete intersection dimension, quasi-projective dimension, and upper Gorenstein dimension. Now it is natural to ask that, can one replace projective dimension by one of these dimensions in (1), (2), or (3)? The main aim of this paper is to give some partial answer to this question. First let us recall the definition of these homological dimensions.

In [5] Avramov, Gasharov, and Peeva has generalized the notion of projective dimension to that of complete intersection dimension and quasi-projective dimension. A diagram of local homomorphisms $R \rightarrow R^{\prime} \leftarrow Q$, with $R \rightarrow R^{\prime}$ a flat extension and $R^{\prime}=Q /(x)$ where $x=x_{1}, x_{2}, \ldots, x_{c}$ is a Q-regular sequence, is called a quasi-deformation of codimension $c$. The complete intersection dimension and quasi-projective dimension of the $R$-module $M$ are denoted by CI- $\operatorname{dim}_{R} M$ and $\operatorname{qpd}_{R} M$, and defined as

\section{CI- $\operatorname{dim}_{R} M$}

$$
=\inf \left\{\operatorname{pd}_{Q}\left(M \otimes_{R} R^{\prime}\right)-\operatorname{pd}_{Q} R^{\prime} \mid R \rightarrow R^{\prime} \leftarrow Q \text { is a quasi-deformation }\right\},
$$

and

$$
\operatorname{qpd}_{R} M=\inf \left\{\operatorname{pd}_{Q}\left(M \otimes_{R} R^{\prime}\right) \mid R \rightarrow R^{\prime} \leftarrow Q \text { is a quasi-deformation }\right\} .
$$

In [16] Velich has generalized the notion of projective dimension to that of upper Gorenstein dimension. An $R$-module $M$ is called perfect if G- $\operatorname{dim}_{R} M=$ $\operatorname{pd}_{R} M$. The ideal $J$ is called Gorenstein ideal (of grade $g$ ) if $R / J$ is perfect as an $R$-module and $\operatorname{Ext}^{g}(R / J, R) \cong R / J$. A local surjection $\pi: Q \rightarrow R$ is called a Gorenstein deformation if $\operatorname{Ker}(\pi)$ is a Gorenstein ideal. A Gorenstein quasi-deformation of $R$ is a diagram of local homomorphisms $R \rightarrow R^{\prime} \leftarrow Q$, with $R \rightarrow R^{\prime}$ a flat extension and $R^{\prime} \leftarrow Q$ a Gorenstein deformation. The upper Gorenstein dimension of the $R$-module $M$ is defined as

$\mathrm{G}^{*}-\operatorname{dim}_{R} M=\inf \left\{\operatorname{pd}_{Q}\left(M \otimes_{R} R^{\prime}\right)-\operatorname{pd}_{Q} R^{\prime} \mid R \rightarrow R^{\prime} \leftarrow Q\right.$

is a Gorenstein quasi-deformation\}. 
It is well-known that for each $R$-module $M$ there are inequalities:

$$
\mathrm{G}^{-\operatorname{dim}_{R}} M \leq \mathrm{G}^{*}-\operatorname{dim}_{R} M \leq \mathrm{CI}-\operatorname{dim}_{R} M \leq \mathrm{pd}_{R} M,
$$

where finiteness of one of them implies equalities to the left of it, cf. [16, Prop. 2.6].

The $n$th Betti number of $M$ over $R$ is defined by $\beta_{n}^{R}(M)=\operatorname{rank}_{k}\left(\operatorname{Ext}_{R}^{n}(M\right.$, $k)$ ). The complexity of $M$ is defined by Avramov [4] as

$\operatorname{cx}_{R} M=\inf \left\{d \in \mathrm{N}_{0} \mid \beta_{n}^{R}(M) \leq a n^{d-1}\right.$ for some positive real $a$ and $\left.n \gg 0\right\}$.

The finiteness of complete intersection dimension implies the finiteness of complexity; this is essentially due to Gulliksen, cf. [10].

In [5, Thm. 5.11] it has been shown that for any $R$-module $M$ there is an equality $\operatorname{qpd}_{R} M=$ CI- $\operatorname{dim}_{R} M+\mathrm{cx}_{R} M$. Therefore the following implications hold:

$$
\operatorname{cx}_{R} M<\infty, \text { and } \mathrm{CI}-\operatorname{dim}_{R} M<\infty \Leftrightarrow \operatorname{qpd}_{R} M<\infty .
$$

In section 2 it is shown that one can replace the upper Gorenstein dimension with the projective dimension in the inequalities (2) and (3), see Theorem 2.1 In addition we show that the grade of a module of finite upper Gorenstein dimension is actually equal to its codimension, see Proposition 2.2.

In section 3 it is shown that the projective dimension can be replaced by the quasi-projective dimension in (1), see Theorem 3.1. By giving an example we show that one can not replace projective dimension with complete intersection dimension in (1). The last result of this paper is a generalization of a result due to Araya and Yoshino, [2, Theorem 3.1], by showing the following, see Theorem 3.3.

Let $M$ and $N$ be finite $R$-modules with $\operatorname{qpd}_{R} N<\infty$ and $\operatorname{Tor}_{i}^{R}(M, N)=0$ for any $i>0$. Then

$\operatorname{grade}_{R}(L, M)-\operatorname{qpd}_{R} N \leq \operatorname{grade}_{R}\left(L, M \otimes_{R} N\right)-\operatorname{cx}_{R} N \leq \operatorname{grade}_{R}(L, M)$.

\section{Upper Gorenstein dimension}

In this section it is shown that the ring $R$ is Cohen-Macaulay if there exists a Cohen-Macaulay finite $R$-module $M$ of finite upper Gorenstein dimension. In addition, it is shown that the grade of a module of finite upper Gorenstein dimension is actually equal to its codimension.

THEOREM 2.1. Let $M$ be a finite R-module with finite upper Gorenstein dimension. Then the following hold

(a) cmd $R \leq \operatorname{cmd}_{R} M$. 
(b) $\operatorname{dim} R \leq \operatorname{dim}_{R} M+\mathrm{G}^{*}-\operatorname{dim}_{R} M$.

Proof. (a) Since $\mathrm{G}^{*}$-dim $M<\infty$, there exists a quasi-deformation $R \rightarrow$ $R^{\prime}=Q / J \leftarrow Q$ with $\operatorname{pd}_{Q} M^{\prime}<\infty$, where $M^{\prime}=M \otimes_{R} R^{\prime}$. By the Intersection Theorem, cmd $Q \leq \mathrm{cmd}_{Q} M^{\prime}$. It is well-known that $\mathrm{cmd}_{Q} M^{\prime}=$ $\operatorname{cmd}_{R^{\prime}} M^{\prime}$. Now since $R \rightarrow R^{\prime}$ is a flat extension, by $[6,1.2]$ the following hold

$$
\begin{aligned}
\operatorname{cmd} R^{\prime} & =\operatorname{cmd} R+\operatorname{cmd}_{R} R^{\prime} / \mathfrak{m} R^{\prime} ; \\
\operatorname{cmd}_{R^{\prime}} M^{\prime} & =\operatorname{cmd}_{R} M+\operatorname{cmd}_{R} R^{\prime} / \mathfrak{m} R^{\prime} .
\end{aligned}
$$

On the other hand by [7, Cor. 3.12] we have cmd $Q=\mathrm{cmd} R^{\prime}$. Therefore

$$
\begin{aligned}
\operatorname{cmd} R+\operatorname{cmd}_{R} R^{\prime} / \mathrm{m} R^{\prime} & =\operatorname{cmd} Q \\
& \leq \operatorname{cmd}_{Q} M^{\prime} \\
& =\operatorname{cmd}_{R} M+\operatorname{cmd}_{R} R^{\prime} / \mathrm{m} R^{\prime} .
\end{aligned}
$$

(b) This part is obtained by applying (a) and the equality depth $R=$ $\operatorname{depth}_{R} M+\mathrm{G}^{*}-\operatorname{dim}_{R} M$, cf. [16, Prop. 2.4].

Let $M$ be a finite $R$-module. The grade of $M$, $\operatorname{grade}_{R} M$, defined by Rees to be the maximal length of $R$-regular sequence in the annihilator of $M$. Also, the codimension $\operatorname{codim}_{R} M$ of the support of $M$ in the spectrum of $R$ is defined as the height of the annihilator of $M$. In [6] Avramov and Foxby have studied some properties of the codimension of a module with finite projective dimension. Now as an application of Theorem 2.1, we give a generalization of [6, Prop. 2.5] for a module with finite upper Gorenstein dimension. Often it is convenient to compute $\operatorname{grade}_{R} M$ and $\operatorname{codim}_{R} M$ from the formulas

$$
\begin{aligned}
\operatorname{grade}_{R} M & =\inf \left\{\operatorname{depth} R_{\mathfrak{p}} \mid \mathfrak{p} \in \operatorname{Supp}_{R} M\right\} ; \\
\operatorname{codim}_{R} M & =\inf \left\{\operatorname{dim} R_{\mathfrak{p}} \mid \mathfrak{p} \in \operatorname{Supp}_{R} M\right\} .
\end{aligned}
$$

Proposition 2.2 (See Prop. 2.5 in [6]). If $M$ is a non-zero finite $R$-module of finite upper Gorenstein dimension, then $\operatorname{grade}_{R} M=\operatorname{codim}_{R} M$ and there exists a prime ideal $\mathfrak{p}$ minimal in $\operatorname{Supp}_{R} M$ such that $R_{\mathfrak{p}}$ is Cohen-Macaulay of dimension grade $_{R} M$.

Proof. Choose $\mathfrak{q} \in \operatorname{Supp}_{R} M$ such that $\operatorname{grade}_{R} M=\operatorname{depth} R_{\mathfrak{q}}$, and then choose $\mathfrak{p}$ contained in $\mathfrak{q}$ and minimal in $\operatorname{Supp}_{R} M$. By using [16, Prop. 2.4] and [16, Prop. 2.10] we conclude from the choices of $\mathfrak{q}$ and $\mathfrak{p}$ that

$$
\begin{aligned}
\operatorname{grade}_{R} M=\text { depth } R_{\mathfrak{q}} \geq \mathrm{G}^{*}-\operatorname{dim}_{R_{\mathfrak{q}}} M_{\mathfrak{q}} \\
\quad \geq \mathrm{G}^{*}-\operatorname{dim}_{R_{\mathfrak{p}}} M_{\mathfrak{p}}=\operatorname{depth} R_{\mathfrak{p}} \geq \operatorname{grade}_{R} M .
\end{aligned}
$$


Therefore $\operatorname{grade}_{R} M=$ depth $R_{\mathfrak{p}}$. Since $M_{\mathfrak{p}}$ is an $R_{\mathfrak{p}}$-module of finite length and of finite upper Gorenstein dimension, the ring $R_{\mathfrak{p}}$ is Cohen-Macaulay by 2.1. Now by the following inequalities

$$
\operatorname{grade}_{R} M=\operatorname{dim} R_{\mathfrak{p}} \geq \operatorname{codim}_{R} M \geq \operatorname{grade}_{R} M,
$$

the assertion holds.

\section{Quasi-projective dimension}

The main result in this section is the Theorem 3.1 that is a generalization of the Intersection Theorem.

THeORem 3.1. Let $M$ be a finite $R$-module with finite quasi-projective dimension. Then for any finite $R$-module $N$,

$$
\operatorname{dim}_{R} N \leq \operatorname{dim}_{R}\left(M \otimes_{R} N\right)+\operatorname{qpd}_{R} M .
$$

Proof. Since $\operatorname{qpd}_{R} M<\infty$, so there is a quasi-deformation $R \rightarrow R^{\prime} \leftarrow Q$ with $\operatorname{pd}_{Q} M^{\prime}<\infty$ where $M^{\prime}=M \otimes_{R} R^{\prime}$. By the Intersection Theorem, cf. [15],

$$
\operatorname{dim}_{Q} N^{\prime} \leq \operatorname{dim}_{Q}\left(M^{\prime} \otimes_{Q} N^{\prime}\right)+\operatorname{pd}_{Q} M^{\prime} .
$$

We have the following

$$
\begin{aligned}
\operatorname{dim}_{Q}\left(M^{\prime} \otimes_{Q} N^{\prime}\right) & =\operatorname{dim}_{R^{\prime}}\left(M^{\prime} \otimes_{R^{\prime}} N^{\prime}\right) \\
& =\operatorname{dim}_{R^{\prime}}\left(M \otimes_{R} N\right)^{\prime} \\
& =\operatorname{dim}_{R}\left(M \otimes_{R} N\right)+\operatorname{dim} R^{\prime} / \mathfrak{m} R^{\prime} .
\end{aligned}
$$

where the last equality holds by [6, Prop. (1.2)]. It is easy to see that

$$
\operatorname{dim}_{Q} N^{\prime}=\operatorname{dim}_{R^{\prime}} N^{\prime}=\operatorname{dim}_{R} M+\operatorname{dim} R^{\prime} / \mathfrak{m} R^{\prime} .
$$

Thus the proof is completed.

The following example shows that we can not replace quasi-projective dimension with complete intersection dimension in Theorem 3.1.

Example 3.2. Let $Q=k\left[\left|x_{1}, x_{2}, \ldots, x_{n}, y_{1}, y_{2}, \ldots, y_{n}\right|\right]$ where $k$ is a field. Let $z_{i}=x_{i} y_{i}$ for $i=1,2, \ldots, n$. Consider the $Q$-ideals $I=\left(z_{1}, z_{2}, \ldots\right.$, $\left.z_{n}\right), J=\left(x_{1}, x_{2}, \ldots, x_{n}\right)$, and $L=\left(y_{1}, y_{2}, \ldots, y_{n}\right)$. Let $R=Q / I$. Then $R$ is complete intersection but is not regular. Consider the $R$-modules $A$ and $B$ as $A=R / J R$ and $B=R / L R$. Then $A \otimes_{R} B=R /(J+L) R$ and hence $\operatorname{dim} A \otimes_{R} B=0$. Since $R$ is complete intersection we have CI- $\operatorname{dim}_{R} A<\infty$ 
and hence by [5, Thm. 1.4], CI- $\operatorname{dim}_{R} A=\operatorname{depth} R-\operatorname{depth}_{R} A=0$. On the other hand $\operatorname{dim} B=n$ so $\operatorname{dim} B>$ CI- $\operatorname{dim}_{R} A+\operatorname{dim} A \otimes_{R} B$.

Let $M$ and $N$ be $R$-modules. Then we define

$$
\operatorname{grade}_{R}(M, N)=\inf \left\{i \mid \operatorname{Ext}_{R}^{i}(M, N) \neq 0\right\} .
$$

If $\operatorname{Ext}_{R}^{i}(M, N)=0$ for all $i$, then $\operatorname{grade}_{R}(M, N)=\infty$. In [2, Theorem 3.1] Araya and Yoshino have used the Intersection Theorem to prove: Let $M$ and $N$ be finite $R$-modules with $\operatorname{pd}_{R} N<\infty$ and $\operatorname{Tor}_{i}^{R}(M, N)=0$ for all $i>0$ then for any finite $R$-module $L$, we have the following inequalities

$$
\operatorname{grade}_{R}(L, M)-\operatorname{pd}_{R} N \leq \operatorname{grade}_{R}\left(L, M \otimes_{R} N\right) \leq \operatorname{grade}_{R}(L, M) .
$$

In the following Theorem we show that, in (4), projective dimension can be replaced by quasi-projective dimension.

Theorem 3.3. Let $M$ and $N$ be finite $R$-modules with $\operatorname{qpd}_{R} N<\infty$ and $\operatorname{Tor}_{i}^{R}(M, N)=0$ for any $i>0$. Then

$\operatorname{grade}_{R}(L, M)-\operatorname{qpd}_{R} N \leq \operatorname{grade}_{R}\left(L, M \otimes_{R} N\right)-\operatorname{cx}_{R} N \leq \operatorname{grade}_{R}(L, M)$.

Proof. By [8, Prop. 1.2.10] there exists $\mathfrak{p} \in \operatorname{Supp}_{R} L \cap \operatorname{Supp}_{R}\left(M \otimes_{R} N\right)$ such that

$$
\operatorname{grade}_{R}\left(L, M \otimes_{R} N\right)=\operatorname{depth}_{R_{\mathfrak{p}}}\left(M_{\mathfrak{p}} \otimes_{R_{\mathfrak{p}}} N_{\mathfrak{p}}\right) .
$$

By [5, Prop. 1.6] CI- $\operatorname{dim}_{R_{\mathfrak{p}}} N_{\mathfrak{p}}<\infty$ and so by applying [12, Thm. 4.3] we have

$$
\operatorname{grade}_{R}\left(L, M \otimes_{R} N\right)=\operatorname{depth}_{R_{\mathfrak{p}}} N_{\mathfrak{p}}+\operatorname{depth}_{R_{\mathfrak{p}}} M_{\mathfrak{p}}-\operatorname{depth}_{R_{\mathfrak{p}}} R_{\mathfrak{p}} .
$$

On the other hand depth $R_{\mathfrak{p}}-\operatorname{depth}_{R_{\mathfrak{p}}} N_{\mathfrak{p}}=$ CI-dim $R_{R_{\mathfrak{p}}} N_{\mathfrak{p}}$, see [5, Thm. 1.4]. Thus grade $_{R}\left(L, M \otimes_{R} N\right)=\operatorname{depth}_{R_{\mathfrak{p}}} M_{\mathfrak{p}}-$ CI-dim $R_{\mathfrak{p}} N_{\mathfrak{p}}$. Therefore by applying [8, Prop. 1.2.10]

$$
\operatorname{grade}_{R}\left(L, M \otimes_{R} N\right) \geq \operatorname{grade}_{R}(L, M)-\mathrm{CI}-\operatorname{dim}_{R} N .
$$

Now the left inequality is obtained by applying the equality CI- $\operatorname{dim}_{R} N=$ $\operatorname{qpd}_{R} N-\operatorname{cx}_{R} N$.

For the right inequality, there exists $\mathfrak{p} \in \operatorname{Supp} M \cap \operatorname{Supp} L$ such that $\operatorname{grade}_{R}(L, M)=\operatorname{depth}_{R_{\mathrm{p}}} M_{\mathfrak{p}}$. Let $\mathfrak{q}$ be a minimal element of the set $\operatorname{Supp}(R /$ $\left.\mathfrak{p} \otimes_{R} M \otimes_{R} N\right)$. Then $\mathfrak{p} \subseteq \mathfrak{q}$ and so $\mathfrak{q} \in \operatorname{Supp} L$. By Theorem 3.1, $\operatorname{dim}_{R_{\mathfrak{q}}}\left(R_{\mathfrak{q}} /\right.$ 
$\left.\mathfrak{p} R_{\mathfrak{q}} \otimes_{R_{\mathfrak{q}}} M_{\mathfrak{q}}\right) \leq \operatorname{qpd}_{R_{\mathfrak{q}}} N_{\mathfrak{q}}$ and hence by [5, Thm. 5.11] $\operatorname{dim}_{R_{\mathfrak{q}}}\left(R_{\mathfrak{q}} / \mathfrak{p} R_{\mathfrak{q}} \otimes_{R_{\mathfrak{q}}}\right.$ $\left.M_{\mathfrak{q}}\right) \leq$ CI- $\operatorname{dim}_{R_{\mathfrak{q}}} N_{\mathfrak{q}}+\operatorname{cx}_{R_{\mathfrak{q}}} N_{\mathfrak{q}}$. The following inequalities hold:

$$
\begin{aligned}
\operatorname{CI}^{-\operatorname{dim}_{R_{\mathfrak{q}}} N_{\mathfrak{q}}} & \geq \operatorname{dim}_{R_{\mathfrak{q}}}\left(R_{\mathfrak{q}} / \mathfrak{p} R_{\mathfrak{q}} \otimes_{R_{\mathfrak{q}}} M_{\mathfrak{q}}\right)-\operatorname{cx}_{R_{\mathfrak{q}}} N_{\mathfrak{q}} \\
& \geq \operatorname{dim}_{R_{\mathfrak{p}}}\left(R_{\mathfrak{p}} / \mathfrak{p} R_{\mathfrak{p}} \otimes_{R_{\mathfrak{p}}} M_{\mathfrak{p}}\right)+\operatorname{dim}_{R_{\mathfrak{q}}} R_{\mathfrak{q}} / \mathfrak{p} R_{\mathfrak{q}}-\operatorname{cx}_{R_{\mathfrak{q}}} N_{\mathfrak{q}} \\
& \geq \operatorname{depth}_{R_{\mathfrak{q}}} M_{\mathfrak{q}}-\operatorname{depth}_{R_{\mathfrak{p}}} M_{\mathfrak{p}}-\operatorname{cx}_{R_{\mathfrak{q}}} N_{\mathfrak{q}} \\
& \geq \operatorname{depth}_{R_{\mathfrak{q}}} M_{\mathfrak{q}}-\operatorname{depth}_{R_{\mathfrak{p}}} M_{\mathfrak{p}}-\operatorname{cx}_{R} N .
\end{aligned}
$$

Therefore

$$
\begin{aligned}
\operatorname{cx}_{R} N+\operatorname{grade}_{R}(L, M) & \geq \operatorname{cx}_{R} N+\operatorname{depth}_{R_{\mathfrak{p}}} M_{\mathfrak{p}} \\
& \geq \operatorname{depth}_{R_{\mathfrak{q}}} M_{\mathfrak{q}}-\text { CI-dim } R_{R_{\mathfrak{q}}} N_{\mathfrak{q}} \\
& =\operatorname{depth}_{R_{\mathfrak{q}}}\left(M_{\mathfrak{q}} \otimes_{R_{\mathfrak{q}}} N_{\mathfrak{q}}\right) \\
& \geq \operatorname{grade}_{R}\left(L, M \otimes_{R} N\right) .
\end{aligned}
$$

Now the assertion holds.

The following example shows that the term " $\operatorname{cx}_{R} N$ " is necessary in the Theorem 3.3.

Example 3.4. Let $R=k[|X, Y|] /(X Y), N=R / y R$ where $x$ (resp. $y$ ) is image of $X$ (resp. $Y$ ) in $R$. Since $R$ is complete intersection so CI-dim ${ }_{R} N<$ $\infty$. Since $\operatorname{depth}_{R} N=1$ we have CI- $\operatorname{dim}_{R} N=0$, the quasi-deformation can be chosen as $R=R^{\prime}$ and $Q=k[|X, Y|]$. Then it is easy to see that $\operatorname{cx}_{R}(N)=1$. Set $M=R$ and $L=R / x R$. Then $\operatorname{grade}_{R}(L, M)=0$ and $\operatorname{grade}_{R}\left(L, M \otimes_{R} N\right)=1$.

AcKnOwLedgments. The authors would like to thank Sean SatherWagstaff for his invaluable comments. Also they would like to thank the referee for his/her useful comments.

\section{REFERENCES}

1. Apassov, D., Almost finite modules, Comm. Algebra 27 (1999), 919-931.

2. Araya, T., Yoshino, Y., Remarks on a depth formula, a grade inequality and a conjecture of Auslander, Comm. Algebra 26 (1998), 3793-3806.

3. Auslander, M., Bridger, M., Stable module theory, Memoirs of the Amer. Math. Soc. 94, American Mathematical Society, Providence, R.I., 1969.

4. Avramov, L. L., Homological asymtotics of modules over local rings, in "Commutative Algebra”, Vol. 15, pp. 33-62, MSRI, Berkeley, 1982, Springer-Verlag, New York, 1989.

5. Avramov, L. L., Gasharov, V. N., Peeva, I. V., Complete intersection dimension, Inst. Hautes Études Sci. Publ. Math. 86 (1997), 67-114. 
6. Avramov, L. L., Foxby, H.-B., Cohen-Macaulay properties of ring homomorphisms, Adv. Math. 133 (1998), 54-95.

7. Avramov, L. L., Foxby, H.-B., Herzog, B., Structure of local homomorphisms, J. Algebra 164 (1994), 124-145.

8. Bruns, W., Herzog, J., Cohen-Macaulay rings, Cambridge Studies in Advanced Mathematics 39, Cambridge University Press, Cambridge, 1993.

9. Foxby, H.-B., Hyperhomological algebra and commutative algebra, Notes in preparation.

10. Gulliksen, T. H., A change of ring theorem with applications to Poincaré series and intersection multiplicity, Math. Scand. 34 (1974), 167-183.

11. Hochster, M., Topics in the homological theory of modules over commutative rings, CBMS Regional Conf. Ser. in Math., vol. 24, Amer. Math. Soc. (1975).

12. Iyengar, S., Depth for complexes, and intersection theorems, Math. Z. 230 (1999), 545-567.

13. Peskine, C., Szpiro, L., Syzygies et multiplicités, C. R. Acad. Sci. Paris Sér. A 278 (1974), 1421-1424.

14. Roberts, P., Two applications of dualizing complexes over local rings, Ann. Sci. École Norm. Sup. (4) 9 (1976), 103-106.

15. Roberts, P., Le théorème d'intersection, C. R. Acad. Sci. Paris Sér. I Math. 304 (1987), 177180

16. Veliche, O., Construction of modules with finite homological dimensions, J. Algebra 250 (2002), 427-449.

17. Yassemi, S., G-dimension, Math. Scand. 77 (1995), 161-174.

SCHOOL OF MATHEMATICS

INSTITUTE FOR STUDIES IN THEORETICAL

PHYSICS AND MATHEMATICS

P.O. BOX 19395-5746

TEHRAN

IRAN
DEPARTMENT OF MATHEMATICS UNIVERSITY OF TEHRAN

TEHRAN

IRAN

E-mail: yassemi@ipm.ir 